\title{
Evolution of a pulse of noninteracting quasiparticles with dispersion and initial angular width
}

\author{
I.N. Adamenko ${ }^{1,2}$, K.E. Nemchenko ${ }^{1}$, V.A. Slipko ${ }^{1}$, and A.F.G. Wyatt ${ }^{3}$ \\ ${ }^{1}$ Karazin Kharkov National University, 4 Svobody Sq., Kharkov 61077, Ukraine \\ E-mail: nemchenko@pht.univer.kharkov.ua \\ ${ }^{2}$ Scientific and Technological Center of Electrophysics, NAN of Ukraine \\ 28 Chernyshevskyi Str., P.O. Box 8812, Kharkov 61002, Ukraine \\ ${ }^{3}$ School of Physics, University of Exeter, Exeter EX4 4QL, UK
}

Received December 18, 2003

\begin{abstract}
The evolution of a pulse of noninteracting quasiparticles, caused by their different velocities and angular distribution of momenta, is studied theoretically. Equations are found that describe the shape of the pulse surface at any time. The time of the beginning, end and duration of the density of the quasiparticle energy flux is determined at a general spatial point. The quasiparticle energy density is considered at all times and positions, and it is shown that the region of high energy density, in the middle of the pulse, is equal to the initial energy density under certain conditions. These theoretical results are discussed in relation to experimental data on the evolution of a pulse of noninteracting phonons in superfluid helium.
\end{abstract}

PACS: 67.40.Fd

\section{Introduction}

Quasiparticle pulses are studied theoretically and experimentally in various fields of modern physics. The variety of phenomena that appear are due to a number of factors, the most important of which is the role played by following:

1. The dependence of the quasiparticle energy on momentum (the dispersion or energy - momentum relation). Classical particles can be considered as quasiparticles with a quadratic energy - momentum relation.

2. The angular width of the pulse, which is determined by the angular range of the quasiparticles' momenta.

3. Interactions between quasiparticles.

4. The interaction of quasiparticles with the medium in which the pulse moves.

The above-mentioned factors determine, for example, phenomena observed in photon pulses propagating in an optically transparent medium with variable refraction index, e.g., optical waveguides, or in a medium where the refractive index depends on photon energy. Another interesting property of anisotropic crystals is phonon focusing, which is caused by pho- nons having different velocities in different directions. Also a large variety of phenomena are observed in pulses of common particles.

Superfluid helium (He II) is a unique medium for considering quasiparticle propagation. This is due to a number of factors.

1. In superfluid helium there exist quasiparticles with different energy - momentum relations, phonons with an almost linear relation, $\mathrm{R}^{+}$-rotons with a relation which is close to quadratic but at a finite energy and momentum, and $\mathrm{R}^{-}$-rotons, whose velocity and momentum have opposite directions. The results of experiments on phonon pulses can be found in $[1,2]$, $\mathrm{R}^{+}$-rotons in [3,4], and $\mathrm{R}^{-}$-rotons in [5].

2 . By changing the temperature of superfluid helium, one can control the interactions of quasiparticles with the ambient medium. So, at relatively high temperatures, a pulse of quasiparticles moving in superfluid helium interacts with the gas of thermal excitations of He II. At relatively low temperatures, when the contribution of the thermal excitation can be neglected, the pulse propagates in a «superfluid vacuum», and its evolution is just determined by the properties of the quasiparticles in the pulse. 
3. Interactions between quasiparticles in superfluid helium can be altered by pressure. The use of pressure allows us to reach two limiting cases: the creation of a pulse of strongly interacting phonons and a pulse of almost noninteracting phonons.

At the saturated vapor pressure, low-energy phonons ( $l$-phonons) with energy $\varepsilon<10 \mathrm{~K}$ have anomalous dispersion [6]. In this case, fast three-phonon processes are allowed and cause an almost instantaneous equilibrium in the system of $l$-phonons that form the pulse. This fast relaxation causes a unique phenomenon; a pulse of low energy-phonons creates high-energy phonons ( $h$-phonons). Their energy $\varepsilon \geq 10 \mathrm{~K}$, is an order of magnitude greater than the temperature of the $l$-phonons. This phenomena was discovered and described in $[7,8]$, and theory of this process was presented in [9-11]. The results of an experimental study of the evolution of strongly interacting phonons was presented in $[12,13]$ and the theory for these pulses was given in [14].

At a pressure of $19 \mathrm{~atm}$, the dispersion of phonons becomes normal [15]. In this case fast three-phonon processes are prohibited by energy and momentum conservation laws, and four-phonon processes are weak. Thus, it is possible to create a pulse of almost noninteracting phonons, that move in a «superfluid vacuum», formed by He II. Results of experiments on the evolution of such pulses formed by noninteracting phonons are presented in $[16,17]$.

The main aim of this paper is the theoretical study of the evolution in time and space of a pulse of noninteracting quasiparticles propagating in vacuum. This is the first step to solving the inverse problem which would enable us to derive the initial characteristics of a phonon pulse, created by a heater, from detected signals at different points in space. The results of this theory are compared to the experimental data of $[16,17]$. It complements the theory of the evolution of a pulse of strongly interacting phonons (see [14]) and can stimulate new experiments. Moreover, the methods presented in this paper can be used to describe the evolution of pulses formed by weakly interacting particles and quasiparticles in other fields of physics.

\section{Description of a pulse of noninteracting quasiparticles}

A pulse of quasiparticles can be described by the quasiparticle distribution function $n(\mathbf{p}, \mathbf{r}, t)$ that determines the number of quasiparticles in a quantum state, which includes the point of phase space $\mathbf{p}, \mathbf{r}$.

An important property of a pulse is its energy density

$$
E(\mathbf{r}, t)=\int_{p_{0}}^{p_{1}} \int_{\Omega_{p}} \varepsilon n \frac{p^{2} d p d \Omega}{(2 \pi \hbar)^{3}},
$$

where $\varepsilon=\varepsilon(p)$ is the energy of the quasiparticle, $\Omega_{p}$ is the solid angle in which the momenta of the quasiparticles lie, and the limits $p_{0}$ and $p_{1}$ determine the momentum interval for the quasiparticles under consideration. However, the signal amplitude measured by a bolometer is determined by the energy flux density $I$ through the area $d \mathbf{a}$, which is equal to the energy passing per unit time through a unit surface area $d \mathbf{a}$ situated at point $\mathbf{r}$ :

$$
I(\mathbf{r}, t)=\int_{p_{0}}^{p_{1}} \int_{\Omega_{p}} \varepsilon \mathbf{v}_{\mathrm{gr}} \mathbf{N}_{\mathrm{a}} n \frac{p^{2} d p d \Omega}{(2 \pi \hbar)^{3}},
$$

where $\mathbf{v}_{\mathrm{gr}}=\partial \varepsilon / \partial \mathbf{p}$ is the group velocity of the quasiparticle, and $\mathbf{N}_{\mathbf{a}}$ is the unit vector of the area $d \mathbf{a}$.

The distribution function $n$, included in (1), (2) can be found from the solution of the kinetic equation

$$
\frac{\partial n}{\partial t}+\mathbf{v}_{\text {gr }} \frac{\partial n}{\partial \mathbf{r}}=J,
$$

where $J$ is the collision integral, which determines the change in the number of quasiparticles per unit time in the quantum state under consideration, caused by their interaction.

The kinetic equation (3) should be supplemented by the initial condition

$$
n(\mathbf{p}, \mathbf{r}, t=0)=n_{0}(\mathbf{p}, \mathbf{r}),
$$

which determines the pulse in its initial state at $t=0$. In experiments this is the time of the end of the heater pulse. Equation (3) together with the initial condition (4) is the complete formulation of the mathematical problem for deriving the function $n(\mathbf{p}, \mathbf{r}, t)$. In the general case such a problem has no analytical solution, since the collision integral $J$ in (3) is a nonlinear integral operator, including distribution functions with different momenta. So various model solutions are used to treat definite physical problems. For example, in [14], a model solution for a phonon system with fast relaxation allowed a complete description of the evolution of a phonon pulse in superfluid helium and gave results which compared with experimental data [12,13].

Here we consider the opposite limiting case, when the interactions between quasiparticles are so weak that their contribution to the evolution of the pulse can be neglected. In this case, the collision integral $J$, in the kinetic equation (3), is equal to zero, and the solution of the problem can be easily found and looks like 


$$
n(\mathbf{p}, \mathbf{r}, t)=n_{0}\left(\mathbf{p}, \mathbf{r}-\mathbf{v}_{\mathrm{gr}} t\right) .
$$

Using this solution we can rewrite (1) and (2) as follows:

$$
\begin{array}{r}
E(\mathbf{r}, t)=\int_{p_{0}}^{p_{1}} \int_{\Omega_{p}} \varepsilon n_{0}\left(\mathbf{p r}-\mathbf{v}_{\mathrm{gr}} t\right) \frac{p^{2} d p d \Omega}{(2 \pi \hbar)^{3}}, \\
I(\mathbf{r}, t)=\int_{p_{0}}^{p_{1}} \int_{\Omega_{p}} \varepsilon \mathbf{v}_{\mathrm{gr}} \mathbf{N}_{\mathbf{a}} n_{0}\left(\mathbf{p}, \mathbf{r}-\mathbf{v}_{\mathrm{gr}} t\right) \frac{p^{2} d p d \Omega}{(2 \pi \hbar)^{3}} .
\end{array}
$$

According to Eqs. (6) and (7) the temporal deformation of the pulse from its initial state is determined by two factors:

1. The finite angular range of the momenta in the pulse, which is defined by the solid angle

$$
\Omega_{p}=2 \pi \zeta_{p}
$$

where

$$
\zeta_{p}=1-\cos \theta_{p}
$$

is the angular-width parameter. If $\zeta_{p} \neq 0$, quasiparticles in the pulse have different directions, and this results in a deformation of the initial pulse.

2. The dispersion, when quasiparticles with different momentum have different group velocities, results in a temporal widening of initial pulse.

If dispersion is neglected, the group velocity can be written in the form $\mathbf{v}_{\mathrm{gr}}=c \mathbf{p} / p$, and it follows from Eqs. (6) and (7) when $n_{0}(p, \mathbf{r})$ that

$$
\begin{gathered}
E(\mathbf{r}, t)=\int_{\Omega_{p}} E_{0}\left(\mathbf{r}-c t \frac{\mathbf{p}}{p}\right) \frac{d \Omega}{\Omega_{p}} \\
I(\mathbf{r}, t)=c \int_{\Omega_{p}} E_{0}\left(\mathbf{r}-c t \frac{\mathbf{p}}{p}\right) \frac{\mathbf{p}}{p} \mathbf{N}_{\mathbf{a}} \frac{d \Omega}{\Omega_{p}}
\end{gathered}
$$

where

$$
E_{0}(\mathbf{r})=E(\mathbf{r}, t=0)=\int_{p_{0}}^{p_{1}} \varepsilon \mathbf{n}_{0}(p, \mathbf{r}) \frac{p^{2} d p \Omega p}{(2 \pi \hbar)^{3}}
$$

is the energy density in the initial pulse.

Equations (10) and (11) have a simple physical meaning which allows us to describe, the temporal and spatial evolution of the phonon pulse without doing the integration. In order to find the energy density and the energy flux density at the point $\mathbf{r}$ and at time $t$ according to Eqs. (10) and (11), one should consider a sphere of radius $c t$ and center at $\mathbf{r}$. The energy density at the point $\mathbf{r}$ is equal to the average value of the ini- tial density on the spherical surface of a segment of this sphere with solid angle $\Omega_{p}$.

By analogy, one can interpret the integrands in the general expressions (6) and (7) as the partial contribution from quasiparticles with momentum $\mathbf{p}$ to the energy $E(\mathbf{r}, t)$ and to the flux $I(\mathbf{r}, t)$.

\section{The shape of the quasiparticle pulse at different times}

Let us consider a pulse, moving along the $z$ axis, with the initial distribution function

$$
n_{0}(\mathbf{p}, r)=\tilde{n}_{0}(p, \mathbf{r}) g(\mathbf{r}) \eta\left(\frac{p_{z}}{p}-\cos \theta_{p}\right),
$$

where

$$
g(\mathbf{r})=\eta\left(L_{\perp}-r\right) \eta\left(L_{\|}-z\right) \eta(z)
$$

where $\eta$ is the Heaviside step function, which is equal to unity or zero when the argument is positive or negative, respectively. $n_{0}(\mathbf{p}, r)$ is nonzero inside the cylinder (14) for all values of momentum, determined by the limits of integration in Eqs. (1) and (2). This distribution function describes the pulse considered below. It consists of quasiparticles whose momenta lie inside a cone with solid angle $\Omega_{p}$, moving along an axis parallel to the $z$ axis of the cylinder (14).

Let us suppose that the moduli of the group velocities of the quasiparticles in this pulse, vary between the maximum value

$$
v_{\max }=c+v_{1}
$$

to the minimum value

$$
v_{\min }=c-v_{2} .
$$

Let us obtain the equation that describes the shape of this pulse at different times. At time $t$, the length of the quasiparticle's path changes from its maximum value

$$
L_{\max }=v_{\max } t
$$

to its minimum value

$$
L_{\min }=v_{\min } t .
$$

At time $t$, the cross section of the pulse which includes the $z$ axis (see Fig. 1) is bounded by the front, back, and side surfaces. These are formed by quasiparicles which were in the corresponding surfaces of the initial pulse.

According to (6), the front and the side surfaces of the pulse at time $t$ are formed by the points $z$ and $r$, which are the centers of segments of spherical surfaces with radius $L_{\max }$ and solid angle $\Omega_{p}$ with axes directed oppositely to the $z$ axis. The spherical surface of 


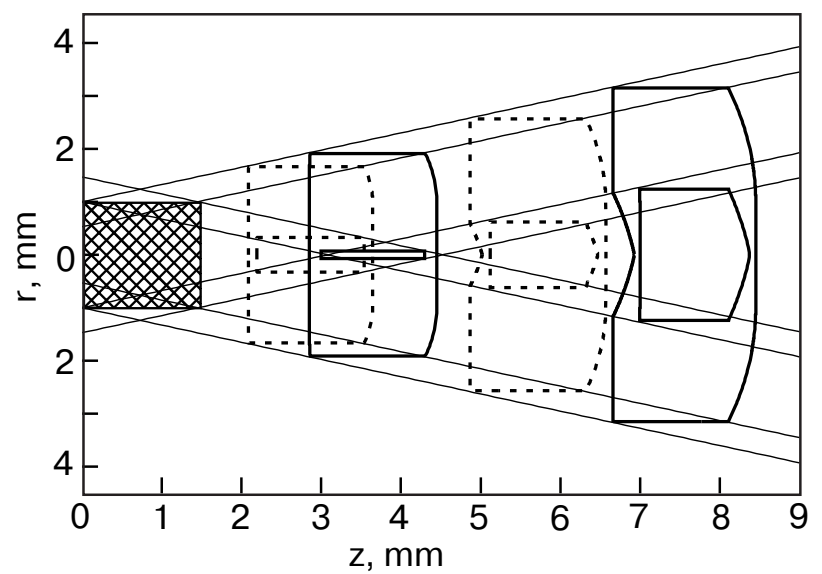

Fig. 1. The shape and form of the structure of the phonon pulse with $t_{p}=4 \mu s$ and $\theta_{p}=18^{\circ}$, and $L_{\perp}=1 \mathrm{~mm}$ at times $t_{1}=8.3 \mu s<t_{\alpha}$ and $t_{2}=19 \mu s>t_{\alpha}$ in the plane of the cross section which includes the axis of the initial pulse. Solid lines represent phonons which move with maximum velocity $v_{\max }=363 \mathrm{~m} / \mathrm{s}$, and dotted lines correspond to the minimum velocity $v_{\min }=265 \mathrm{~m} / \mathrm{s}$. At time $t_{1}$, the rectangular region, in the center of the pulse, has the initial value of the energy density for phonons with a given velocity. The intersection of all these regions gives the region with the initial value of the energy density. At time $t_{2}$ the region in the center of the pulse has a relatively high energy density, but the dispersion has already led to its decrease.

a segment should not intersect the front surface of initial pulse but just have a common point with the initial front surface. A plane section through the symmetry axis is shown in Fig. 1. In other words, the points that form the front and side surfaces are at the maximum distance $L_{\max }$ from the initial pulse.

As a result, when $r$ varies between the limits

$$
0 \leq r \leq L_{\perp}
$$

the front surface is the vertical plane with coordinate

$$
z_{F}=L_{\|}+L_{\max } .
$$

As $r$ increases in the range:

$$
L_{\perp} \leq r \leq r_{F}(t)
$$

where

$$
r_{F}(t)=L_{\perp}+L_{\max } \sin \theta_{p},
$$

the front surface becomes an arc, which is described by the equation

$$
\left(z-L_{\|}\right)^{2}+\left(r-L_{\perp}\right)^{2}=L_{\max }^{2} .
$$

This equation determines the $r$ and $z$ coordinates of the front surface of the pulse.

At $r=r_{F}$ the side surface is a cylinder of radius $r_{F}$, with front surface at $z=L_{\|}+L_{\max } \cos \theta_{p}$ and back surface at $z=L_{\max } \cos \theta_{p}$.
At points $r>r_{F}$ the signal is equal to zero. Here it should be noted that the second term in Eq. (22) describes the widening of the pulse caused by its finite angular width.

According to (6), the back surface of the pulse at time $t$ is defined by the points $z$ and $r$, which are the centers of segments of spherical surfaces with radius $L_{\min }$ and solid angle $\Omega p$ with axes directed oppositely to the $z$ axis. The spherical surface of this segment should intersect the back surface of the initial pulse and the segment should include the maximum volume of the initial pulse. In this case, the points that form the back surface are at the minimum distance from the back surface of the initial pulse, for a given $L_{\min }$; see Fig. 1 .

As a result, at

$$
t<t_{\alpha}=\frac{z_{\alpha}}{v_{\min } \cos \theta_{p}},
$$

where

$$
z_{\alpha}=L_{\perp} / \tan \theta_{p}
$$

(see at Fig. 1 pulse at $t=t_{1}$ ), the back surface is the vertical plane with the coordinates

$$
z_{B}=L_{\mathrm{min}} \cos \theta_{p},
$$

and the coordinates $r$ are confined by the following inequality:

$$
0 \leq r \leq r_{B}(t)
$$

where

$$
r_{B}(t)=L_{\perp}+L_{\min } \sin \theta_{p} .
$$

At the time $t>t_{\alpha}$ (see pulse at $t=t_{2}$ in Fig. 1) the back surface is the vertical plane with coordinates $z_{B}$, if the coordinates $r$ are confined by the following inequality

$$
r_{a}(t) \leq r \leq r_{B}(t),
$$

where

$$
r_{a}(t)=L_{\min } \sin \theta_{p}-L_{\perp} .
$$

For smaller values of $r$, in the range

$$
0 \leq r \leq r_{a}(t),
$$

the back surface becomes an arc and is described by the equation

$$
z^{2}+\left(r+L_{\perp}\right)^{2}=L_{\min }^{2} .
$$

This surface connects the points $r=r_{a}, z=z_{B}$ with the points

$$
r=0 ; \quad z=\sqrt{L_{\min }^{2}-L_{\perp}^{2}} .
$$

The points of the back vertical surface with coordinates $r=r_{B}, z=z_{B}$ are connected with the end of the 
side face, which has the following coordinates: $r=r_{M}=L_{\perp}+L_{\max } \sin \theta_{p}, z=z_{M}=L_{\max } \cos \theta_{p}$ by a cone-like surface formed by the dispersion.

The length of the pulse $L_{z}(t, r)$, at different $t$ at various $r$, is given by the relation

$$
L_{z}(t, r)=z_{\text {front }}(t, r)-z_{\text {back }}(t, r) .
$$

At $t<t_{\alpha}$ when $r$ is changing between the limits (19), using the relations (20), (26), and (34) we get

$$
L_{z}\left(t<t_{\alpha}, 0 \leq r \leq L_{\perp}\right)=L_{\|}+\left(v_{1}+v_{2}\right) t+c t \zeta_{p}-v_{2} t \zeta_{p} .
$$

The second term on the right-hand side of this equation describes the widening of the pulse caused by dispersion, the third term is caused by the finite angular width of the pulse, and the fourth term is due to the product of dispersion and finite angular width.

At $t>t_{\alpha}$ and $r=0$ from relations (20), (33) and (34) we obtain

$$
\begin{gathered}
L_{z}\left(t>t_{\alpha}, r=0\right)=L_{\|}+\left(v_{1}+v_{2}\right) t+ \\
+L_{\min }\left(1-\sqrt{\frac{L_{\perp}^{2}}{L_{\min }^{2}}}\right) .
\end{gathered}
$$

At $L_{\perp}^{2} / L_{\min }^{2}<<1$ in the third term of the right-hand side of Eq. (36) one can extract the contribution due to the finite angular width

$$
\begin{gathered}
L_{z}\left(t>t_{\alpha}, r=0\right)=L_{\|}+\left(v_{1}+v_{2}\right) t+ \\
+\frac{L_{\perp}^{2}}{2 c t}+\frac{L_{\perp}^{2}}{2 c t}\left(\frac{c}{v_{\text {min }}}-1\right) .
\end{gathered}
$$

Comparing the results of (35) with (36) and (37) it follows that at any time, the widening of the pulse caused by dispersion, grows. The influence of a finite angular width has the opposite effect (near the axis of the pulse). At $t<t_{\alpha}$ the length of the pulse grows linearly, and at $t>t_{\alpha}$ the pulse width, caused by the finite angular width, begins to decrease as $1 / t$.

\section{The energy density of a phonon pulse}

The energy density of a phonon pulse at different points in space and time is determined by the initial phonon distribution function (4). Let us consider the case when the initial distribution (5) is given in the following form:

$$
n_{0}(\mathbf{p}, \mathbf{r})=n_{0}(p) g(\mathbf{r}) \eta\left(\frac{p_{z}}{p}-\cos \theta_{p}\right) .
$$

In order to simplify this problem we will not take dispersion into account. Then the energy density at dif- ferent points in space and time can be calculated by Eq. (6) taking into account (38), can be rewritten as follows:

$$
E(\mathbf{r}, t)=\int_{\Omega_{p}} E_{0} g\left(\mathbf{r}-c t \frac{\mathbf{p}}{p}\right) \frac{d \Omega}{\Omega_{p}},
$$

where

$$
E_{0}=\int_{p_{0}}^{p_{1}} \varepsilon n_{0}(p) \frac{p^{2} d p \Omega p}{(2 \pi \hbar)^{3}}
$$

is the initial energy density.

According to Eq. (39), in order to find the energy density at the point $\mathbf{r}$ at time $t$ one should build a circular cone with apex at the point $\mathbf{r}$ and radius

$$
L_{c}=c t
$$

solid angle $\Omega_{p}$ and with a spherical end cap of radius $L_{c}$. The axis is directed oppositely to the $z$ axis. Below, we refer to this circular cone as simply the cone.

The energy density at the center of the pulse is determined by that part of the spherical surface which lies inside the initial pulse (38). If it lies completely inside the initial pulse, then, according to Eq. (39), the energy density at the point of the apex of this segment is equal to the initial value of the energy density (40). If the spherical surface includes no points of the initial pulse, then the energy density at the apex is zero.

This graphical method of explanation helps us to consider the distribution of the energy. At times $t<t_{\alpha}$ (see pulse at $t=t_{1}$ on Fig. 1) the energy density has a maximum value inside the circle with coordinates

$$
z_{R}=L_{\|}+L_{c} \cos \theta_{p}, \text { and } 0 \leq r \leq r_{a}\left(z_{R}\right),
$$

where

$$
r_{a}(z)=L_{\perp}\left(1-\frac{z-L_{\|}}{z_{\alpha}}\right)
$$

for the case

$$
L_{\|}>L_{c} \zeta_{p}
$$

The spherical surfaces of the cones whose apexes are situated on the circle (42) are found to lie completely inside the initial pulse, and so the energy density on the circle (42) is equal to the initial value.

It should be noted that according to Eq. (35), when the inequality (44) is valid, the length of the initial pulse is greater than its longitudinal broadening caused by the finite angular width. In this case, according to inequality (44), there exists a region inside the pulse, in which the energy density is maximal and equal to the value of the energy density in the ini- 
tial pulse (40) (see cylindrical region at $t=t_{1}$ on Fig. 1). This region is closed by two circles at each end, one of which is given by Eq. (42) and another is given by the following expressions:

$$
z_{L}=L_{c} \text {, and } 0 \leq r \leq r_{a}\left(z_{R}\right) .
$$

The side surface is described by the equation $r=r_{a}\left(z_{R}\right)$.

The length of this region is

$$
z_{R}-z_{L}=L_{\|}-L_{c} \zeta_{p},
$$

and the length of the pulse at this time is equal to

$$
L_{z}=L_{\|}+L_{c} \zeta_{p} .
$$

As a result, the region with the maximum value of energy density, $E_{0}$, is separated from the front and back surfaces by the distance $L_{c} \zeta_{p}$, which is equal to the widening of the pulse. During the motion of the pulse along the $z$ axis, the size of this region with $E_{0}$ shows a monotonic decrease and disappears at the point $z=L_{\|}+z_{\alpha}$, which is the point of intersection of the lines $r_{a}(z)$ with the $z$ axis.

Here we should note that according to [14] a region of the pulse with density $E_{0}$ exists in the opposite limiting case, when the pulse is formed by strongly interacting phonons. However, the physical reasons for this region and its decrease are quite different in the two cases. Moreover, a phonon pulse of strongly interacting phonons, with the initial distribution function (38), moves along the axis $z$ with much less transverse broadening than for noninteracting particles, in spite of the angular width and dispersion (see [14]).

At $t>t_{\alpha}$, the integral (39) always has a region which gives no contribution to the energy density, i.e., where the initial pulse is absent (see pulse at $t=t_{2}$ in Fig. 1). That is why at times $t>t_{\alpha}$, the energy density, at all points in the pulse, is less than the initial energy density (40).

However, in this case the pulse has a region where the energy density is greater than at other points in the pulse. This region has coordinates

$$
z_{R}^{\prime}=L_{\|}+\sqrt{L_{c}^{2}-L_{\perp}^{2}} \text { and } r=0,
$$

since the spherical surface of the cone, with apex at the point (48), intersects the edges of the front surface of the initial pulse at the point with coordinates $z=L_{\|}$and $r=L_{\perp}$. Clearly this region contributes most to the integral (39).

The situation corresponding to inequality (44) in the case considered case takes place at

$$
L_{\|}>L_{c}\left(1-\sqrt{1-\frac{L_{\perp}^{2}}{L_{c}^{2}}}\right),
$$

when the length of initial pulse is greater than the widening caused by finite angular width, described by the third term in right-hand side of Eq. (36).

When the inequality (49) is satisfied, the pulse at $t>t_{\alpha}$ has a relatively high energy density, separated by the surfaces described by Eq. (48) and the point

$$
z_{L}^{\prime}=L_{c} \text { and } r=0
$$

which is the apex of the cone whose spherical surface intersects the side face of the initial pulse and touches its back face.

\section{The beginning, end, and duration of the quasiparticle pulse at different spatial points}

Consider the pulse at $t=0$, described at the beginning of Sec. 3. Taking into account the symmetry of the initial pulse, it is sufficient to consider the solution of the problem in the plane of the cross section which includes the $z$ axis, and coincides with the axis of the cylinder (14), which describes the surface boundaries of the initial pulse.

In experiments, the signals are detected with detectors which are oriented so that all the quasiparticles in the pulse have

$$
\mathbf{v}_{\mathrm{gr}} \mathbf{N}_{\mathbf{a}} \neq 0 .
$$

So, below we will consider the condition (51) to be valid.

The time of the start of the signal $t_{\text {beg }}(z, r)$ detected at the point with coordinates $z$ and $r$ can be found from the geometric interpretation of formula (6) in the way we now describe. One should again construct a cone with apex at the point $z, r$ and axis directed oppositely to axis $z$, with solid angle $\Omega_{p}$. The surface of the cone's spherical end cap should have just one common point with the front or side surfaces of the initial pulse. Then the radius of this segment is calculated and this value gives us the minimum value $R_{\min }(z, r)$, determined by position of point $z, r$. The time of the start of the signal is given by the following formula:

$$
t_{\text {beg }}(z, r)=\frac{R_{\min }(z, r)}{v_{\max }} .
$$

Using this interpretation, the dependence on coordinates $z$ at any $r$ is given by

$$
t_{\text {beg }}\left(z, 0 \leq r \leq L_{\perp}\right)=\frac{z-L_{\|}}{v_{\max }} .
$$

The signals at time (53) is formed by phonons moving parallel to $z$ axis with velocity $v_{\max }$ from the front surface of the initial pulse. On Fig. 1 at $t=t_{1}$ and $t=t_{2}$ these phonons create the front surface. 
Figure 2 shows the dependence on time of the signals at points with different values of $r$ with a fixed value of $z$. In Fig. 2 the time (53) at $z=z_{1}$ and $z=z_{2}$ is shown by the vertical lines which are determined by the interval (19).

On further increasing $r$, when

$$
L_{\perp} \leq r \leq r_{F}(z)
$$

where

$$
r_{F}(z)=L_{\perp}+\left(z-L_{\|}\right) \tan \theta_{p}=L_{\perp} \frac{z+z_{\alpha}-L_{\|}}{z_{\alpha}} .
$$

The time of the start of the signal is given by

$$
\begin{gathered}
t_{\text {beg }}\left(z, L_{\perp} \leq r \leq r_{F}(z)\right)= \\
=\frac{1}{v_{\text {max }}} \sqrt{\left(r-L_{\perp}\right)^{2}+\left(z-L_{\|}\right)^{2}} .
\end{gathered}
$$

The signal at time (56) is formed by quasiparticles that move at a definite angle $0 \leq \theta \leq \theta_{p}$ with respect to the $z$ axis, with velocity $v_{\max }$ from the point $z=L_{\|}$and $r=L_{\perp}$ of the initial pulse. Figure 1 shows that at $t=t_{1}$ and $t=t_{2}$ these quasiparticles create the arc on the front surface of the pulse (23). In Fig. 2 at $z=z_{1}$ and $z=z_{2}$, the time (56) is shown by the curve (56).

And, finally, when $r$ lies between the limits

$$
r_{F}(z) \leq r \leq r_{B}(z),
$$

where

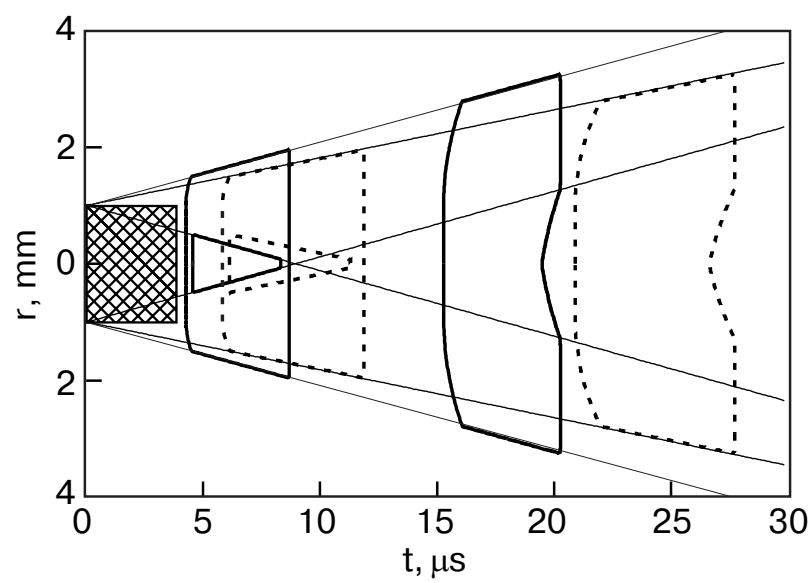

Fig. 2. The beginning, end, and duration of the same phonon signal as in Fig. 1 at points with coordinates $z_{1}<z_{\alpha}$ and $z_{2}>z_{\alpha}$ at different values of the radius $r$. Solid lines represent phonons, moving with maximum velocities, and dotted lines, with minimum velocities. The cone-like region at $z=z_{1}=3 \mathrm{~mm}$ represents the maximum possible amplitude of the signal. At point $z=z_{2}=7 \mathrm{~mm}$ there is no region of constant signal.

$$
r_{B}(z)=L_{\perp}+z \tan \theta_{p}=L_{\perp} \frac{z+z_{\alpha}}{z_{\alpha}},
$$

and the time of the signal start is given by

$$
t_{\text {beg }}\left(z, r_{F}(z) \leq r \leq r_{B}(z)\right)=\frac{r-L_{\perp}}{v_{\max } \sin \theta_{p}} .
$$

The signals at time (59) are formed by quasiparticles, which move at angle $\theta_{p}$ with respect to $z$ axis with the velocity $v_{\max }$ from the side surface of the initial pulse. In Fig. 1 at $t=t_{1}$ and $t=t_{2}$ these quasiparticles form the cylindrical side surface $r=r_{F}(t)$ (see Eq. (22) and the second sentence after Eq. (23)). Figure 2 at $z=z_{1}$ and $z=z_{2}$ shows the time (59) by the segment of line inside the interval (59).

At $r>r_{B}(z)$ there is no quasiparticle signal because $\theta_{p}$ is the maximum angle of quasiparticle motion in the initial pulse.

Using the geometric interpretation of expression (6), let us find the time $t_{\text {end }}(z, r)$ of the end of the signal at a definite point $z, r$. In order to do this, it is sufficient to create the same cone as before but now this segment must intersect the back face of the initial pulse and include as many points of the initial pulse as possible. Then we find the radius of this segment $R_{\max }(z, r)$ and calculate the desired time:

$$
t_{\text {end }}(z, r)=\frac{R_{\max }(z, r)}{v_{\min }} .
$$

Using this approach, for points with $z<z_{\alpha}$, when the radius changes inside the interval

$$
0 \leq r \leq r_{B}(z),
$$

we find the maximum value for the time of the signal end, as will be shown below,

$$
t_{\mathrm{end}}^{(\max )}=\frac{z}{v_{\min }\left(1-\zeta_{p}\right)} .
$$

The signal at time (62) is created by quasiparticles which move at the angle $\theta_{p}$ with respect to the $z$ axis, with velocity $v_{\min }$, from the back surface of the initial pulse. In Fig. 1, at $t=t_{1}$, these phonons form the back surface of the pulse. Figure 2 , at $z=z_{1}$, shows the time (62) by the segment of the vertical line inside the interval (61).

In Fig. 2 at $z=z_{1}$ and $z=z_{2}$ an imaginary horizontal line connects the end of the line describing the beginning of the signal (59) with the end of the vertical line, which describes the time of the end of the signal. These correspond to $v=v_{\max }$ and $v=v_{\min }$. In Fig. 1 at $t=t_{1}$ and $t=t_{2}$ the corresponding construction connects the ends of the back vertical surfaces. 
At $z>z_{\alpha}$, when the radius changes inside the interval

$$
r_{A}(z) \leq r \leq r_{B}(z)
$$

where

$$
r_{A}(z)=L_{\perp} \frac{z-z_{\alpha}}{z_{\alpha}},
$$

the time of the signal end is given by formula (62) and this signal is formed by the same quasiparticles as those at the end of the pulse at $z=z_{1}$. In Fig. 1 at $t=t_{2}$ these quasiparticles form the back surface of the pulse. In Fig. 2 at $z=z_{2}$, the time (62) is shown by the segment of the right vertical line, which is limited by inequalities (63).

At $z>z_{\alpha}$, when the radius changes inside the interval

$$
0 \leq r \leq r_{A}(z),
$$

the time of the end of the signal is given by

$$
t_{\text {end }}\left(z>z_{\alpha}, 0 \leq r \leq r_{A}(z)\right)=\frac{1}{v_{\text {min }}} \sqrt{z^{2}+\left(r+L_{\perp}\right)^{2}} .
$$

At this time, signals are created by quasiparticles that move at a definite angle $0 \leq \theta \leq \theta_{p}$ with respect to the $z$ axis with velocity $v_{\min }$, from the point $z=0, r=L_{\perp}$ of the initial pulse. In Fig. 1 at $t=t_{2}$ these quasiparticles form the arc of the back surface, and in Fig. 2 at $z=z_{2}$, the time (66) is described by the right arc of a hyperbola.

The duration of the pulse at the point $z, r$ is determined by the equality

$$
\Delta t(z, r)=t_{\text {end }}(z, r)-t_{\text {beg }}(z, r) .
$$

According to (53), (56), (59), and (60) at $z<z_{\alpha}$ the duration of the pulse has a maximum value, when $r$ is inside the interval $0 \leq r \leq L_{\perp}$ and equal to

$$
\Delta t_{\max }=t_{p}+z \frac{v_{1}+v_{2}}{v_{\max } v_{\min }}+z \frac{\zeta_{p}}{v_{\min }\left(1-\zeta_{p}\right)},
$$

where $t_{p}=L_{\|} / v_{\max }$ is the initial length of the pulse. In the right-hand side of Eq. (68) the second term describes the increase in pulse length due to dispersion, and the third term is caused by the finite angular width.

Using relations (56), (62), and (67) at $z<z_{\alpha}$ when $L_{\perp} \leq r \leq r_{F}$ the pulse duration is given by

$$
\begin{gathered}
\Delta t_{F}=\Delta t_{\max }+\frac{1}{v_{\max }}\left(z-L_{\|}-\right. \\
\left.-\sqrt{\left(z-L_{\|}\right)^{2}+\left(r-L_{\perp}\right)^{2}}\right) .
\end{gathered}
$$

At $r=L_{\perp}$ the increase in the pulse length, according to (69), reaches its maximum value at $\Delta t_{\max }$. Increasing $r$ causes the signal length to decrease so, that at $r=r_{F}$ we have

$$
\Delta t_{F}\left(r=r_{F}\right)=\frac{t_{p}}{1-\zeta_{p}}+\frac{z}{1-\zeta_{p}} \frac{v_{1}+v_{2}}{v_{\max } v_{\min }} .
$$

According to Eqs. (59), (62), and (67) at $z<z_{\alpha}$ when $r_{F} \leq r \leq r_{B}$, the pulse length is given by

$$
\Delta t_{B}=\frac{z}{v_{\min } \cos \theta_{p}}-\frac{r-L_{\perp}}{v_{\max } \sin \theta_{p}} .
$$

From this equation it follows that the length of the pulse decreases from the maximum value (70) to the minimum value at $r=r_{B}$ and is given by

$$
\Delta t_{B}\left(r=r_{B}\right)=\frac{z}{1-\zeta_{p}} \frac{v_{1}+v_{2}}{v_{\max } v_{\min }},
$$

where the length of the signal does not depend on $t_{p}$ and is determined by the dispersion together with an additional contribution from the finite value of the angular width. At $r>r_{B}$ there is no signal. Expressions (68)-(72) give the length of the pulse at $z<z_{\alpha}$ for all $r$.

At $z>z_{\alpha}$, from Eqs. (53), (66), and (67), for a value of $r$ inside the interval

$$
0 \leq r \leq \min \left(L_{\perp}, r_{A}\right),
$$

it follows that

$$
\Delta t_{\perp}=t_{p}+z \frac{v_{1}+v_{2}}{v_{\max } v_{\min }}+\frac{z}{v_{\min }}\left(\sqrt{1+\left(\frac{r+L_{\perp}}{z}\right)^{2}}-1\right) .
$$

At $r_{A}<L_{\perp}$ expression (74) has a maximum value (68), when $r$ is changing inside the interval $r_{A} \leq r \leq L_{\perp}$.

At $z>z_{\alpha}$ and $r_{A}<L_{\perp}$, when $L_{\perp} \leq r \leq r_{F}$, the length of the pulse can be found from expression (69), and for greater $r\left(r_{F} \leq r \leq r_{B}\right)$ by expression (71).

Using the equations (56), (62), and (66) at $z<2 z_{\alpha}$, for $r$ inside the interval $L_{\perp} \leq r \leq r_{A}$ for the pulse length we get

$\Delta t_{A}=t_{p}+z \frac{v_{1}+v_{2}}{v_{\max } v_{\min }}+\frac{\left(r+L_{\perp}\right)^{2}}{2 z v_{\min }}-\frac{\left(r-L_{\perp}\right)^{2}}{2\left(z-L_{\|}\right) v_{\max }}$.

On the right-hand side of this equation, the third and the fourth terms describe the contribution of the finite angular width, which decreases with increasing $z$. 
At $z>2 z_{\alpha}$ and $L_{\perp}<r_{A}<r<r_{F}$ the length of the signal is given by Eq. (69), and at $r_{F} \leq r \leq r_{B}$, by the relation (71). At $r>r_{B}$ there is no signal.

The result (68)-(75) describes the length of the pulse at any value of $r$ and $z$. From an examination of these results, it follows that the length of the pulse always grows linearly due to dispersion. As to the contribution of the finite angular width, at first (when $z<z_{\alpha}$ and $0 \leq r \leq L_{\perp}$ ) the signal length grows linearly with $z$. At $z>z_{\alpha}$ such a dependence takes place only at $z_{\alpha}<z<2 z_{\alpha}$ and $r_{A} \leq r \leq L_{\perp}$. In all other cases, the contribution of the finite angular width to the increase of the pulse length is either constant (or grows when $\zeta_{p}=0-$ see (70)), or decreases with increasing $z$. For large $z$ the contribution of the finite angular width to the increase of pulse length can be neglected (see (74), (75)).

However, it should be emphasized that according to Eq. (58) it is the finite angular width that always leads to the linear increase of the transverse width of the pulse with increasing $z$. As energy is conserved, this results in the signal decreasing monotonically along the $z$ axis, during the motion of the signal.

\section{Temporal dependence of the signal amplitude in space}

Let us consider the case when the initial distribution is given by relation (38) and omit dispersion, which is not so important for the signal amplitude. In this case relation (7) can be written as

$$
I(\mathbf{r}, t)=c \int_{\Omega_{p}} E_{0} g\left(\mathbf{r}-c t \frac{\mathbf{p}}{p}\right) \frac{\mathbf{p}}{p} \mathbf{N}_{\mathbf{a}} \frac{d \Omega_{p}}{\Omega_{p}} .
$$

The temporal dependence of the signal amplitude at the point $z, r$ can be obtained from the geometrical interpretation of the formula (76) which we used above. To find the amplitude at the point $z, r$ we create a cone with its apex at this point, with solid angle $\Omega_{p}$ and radius $R$, and with its axis directed oppositely to the $z$ axis. The signal amplitude at the apex is determined by the averaged value of (76) using the initial energy density (40). The time $t(R, z, r)$ of the signal, detected at the considered point $z, r$, is determined by

$$
t(R, z, r)=R / c .
$$

If all of the spherical-end surface of the cone lies inside the initial pulse, then, according to (76) at time (77) the signal amplitude is equal to the initial value which would be detected by the bolometer at $t=0$, with the given $\mathbf{N}_{\mathbf{a}}$, situated inside the initial pulse. If the curved surface of the cone has no points within the initial pulse, the signal at time (77) is equal to zero.
Using this approach we can find, without integration, the point $z, r$ at which the amplitude will have maximum value and find the time interval when the maximum value will be observed.

During the initial stage of the evolution, there exists, for a definite time, a region (denoted by the subscript «spot») where the amplitude is equal to its initial value. It follows from (76) at time $t$ that this region is limited by two planes

$$
z_{L}=c t \leq z \leq z_{R}=L_{\|}+c t \cos \theta_{p}
$$

and the cylindrical surface which describes the right-hand side of the inequality

$$
0 \leq r \leq r_{a}\left(z_{R}\right)=L_{\perp}-c t \sin \theta_{p} .
$$

From inequalities (78) and (79), we see that the time duration that this region exists is determined as follows:

$$
t \leq t_{\mathrm{spot}}=\min \left(\frac{L_{\perp}}{c \sin \theta_{p}}, \frac{L_{\|}}{c\left(1-\cos \theta_{p}\right)}\right) .
$$

At $t \leq t_{\text {spot }}$ the region lies within the pulse. In the case of a sufficiently long pulses, for which

$$
\frac{L_{\|}}{1-\cos \theta_{p}}>\frac{L_{\perp}}{\sin \theta_{p}}
$$

the maximum values of $z$ where one can observe the maximum amplitude according to inequalities (78) and (80) is equal to

$$
z_{\max }=z_{R}\left(t_{\text {spot }}\right)=L_{\|}+L_{\perp} \cos \theta_{p}=L_{\|}+z_{\alpha} .
$$

In the case of sufficiently short pulses

$$
\frac{L_{\|}}{1-\cos \theta_{p}}<\frac{L_{\perp}}{\sin \theta_{p}}
$$

from the inequalities (78) and (80), we get

$$
z_{\max }=z_{R}\left(t_{\mathrm{spot}}\right)=\frac{L_{\|}}{1-\cos \theta_{p}} .
$$

The evolution of the maximum-region for the case (81) is presented in the Figs. 1, 2.

In the case (81), at fixed value $z=z_{1}<z_{\max }$ in the plane $r, t$ (see Fig. 2) the high energy density region, with the initial value of the signal amplitude, is determined by the inequalities (see inequalities (78))

$$
\frac{z_{1}-L_{\|}}{c \cos \theta_{p}} \leq t \leq \frac{z_{1}}{c}
$$

and limited by the conical side surface (see inequality (79)) 


$$
r=L_{\perp}-c t \sin \theta_{p} .
$$

When $z>z_{\max }$ the high energy density region is absent and there is no region where amplitude of the signal is constant (see Fig. 2).

\section{Discussion}

The results obtained above allow us to discuss the experimental data presented in [16]. In these experiments the amplitude of phonon signal was measured by a bolometer, situated at the distance $z_{B}=8 \mathrm{~mm}$.

The helium had such a low temperature $\left(T_{\mathrm{He}} \leq 0.1 \mathrm{~K}\right)$ that the thermal excitations can be neglected. A heater injects a pulse of phonons into this pure and isotropic superfluid helium («the superfluid vacu$u m »)$. The duration of the pulse was $5 \mu$ and it moved in the direction normal to the surface of the heater.

The helium was under $24 \mathrm{~atm}$ pressure at which phonon interactions are determined by the relatively slow four-phonon processes. This fact justified the authors using a model of noninteracting phonons, moving ballistically. We start from this model, as it allows us to use the results obtained in this paper.

Here we present the numerical values of all parameters that describe experiments [16]. The sound velocity in helium at pressure of $24 \mathrm{~atm}$ is equal to $c=363 \mathrm{~m} / \mathrm{s}$. The heater was made from a gold film and had dimensions $L_{\perp}=0.5 \mathrm{~mm}$. The velocity of longitudinal sound in the gold is equal to $c_{l}=3240 \mathrm{~m} / \mathrm{s}$ and transverse sound, $c_{t}=1200 \mathrm{~m} / \mathrm{s}$.

Using the acoustical theory of transmission $[18,19]$, we find the maximum value $\theta_{0}$ for a phonon radiated by a perfectly plane heater into helium:

$$
\theta_{0}=\arcsin c / c_{t}=0.307 \mathrm{rad} .
$$

Below, we use this numerical value of the parameter $\theta_{p}=\theta_{0}$ even though the evaporated gold film is rough and the conditions for a critical cone are not met. However, the phonon emission from a gold film has been measured and is found to be strongly peaked in the direction of the surface normal [20]. Then for typical distance $z_{\alpha}$, according to (25) we have $z_{\alpha}=1.58 \mathrm{~mm}$, and for the length of the initial pulse $L_{\|}=t_{p} c$ in this case we have $L_{\|}=1.82 \mathrm{~mm}$. The size of bolometer was $1 \times 1 \mathrm{~mm}$.

The amplitude of the phonon signal was measured at distance $z_{B}$, which is five times more than $z_{\alpha}$. In this case we will use expression (74) for calculating the pulse length.

To calculate the velocities $v_{1}$ and $v_{2}$, included to (74), we use the energy - momentum relation for phonons

$$
\varepsilon=c p\left(1-\gamma p^{2}\right),
$$

where, according to [16], at a pressure of $24 \mathrm{~atm}$, the dispersion parameter is equal to $\gamma=8.96 \cdot 10^{-4} \mathrm{~K}^{-2}$. According to (87) one can consider that $v_{1}=0$ see (15), (16), and $v_{2}$ can be found from the relation

$$
v_{2}=\overline{\left(c-v_{\text {gr }}\right)},
$$

where the line denotes averaging with respect to momentum with an equilibrium function for the initial phonons in the pulse. Integration of (88) with the Bose-Einstein distribution function gives

$$
\frac{v_{2}}{c}=0.028 T_{0}^{2},
$$

where $T_{0}$ is the temperature that describes the distribution of the initial phonon pulse.

Comparing the numerical values of the second and the third terms in right-hand side of Eq. (74), which describe the broadening of the pulse caused respectively by dispersion and the finite angular width of the initial pulse, we conclude that the contribution of the latter is negligibly small. The numerical value of the contribution of the dispersion is equal to

$$
\delta t_{\text {disp }}=\frac{z_{B}}{c} \frac{v_{2}}{v_{\min }}=\frac{0.62 T_{0}^{2}}{1-0.028 T_{0}^{2}} \mu \mathrm{s} .
$$

According to the result presented in Sec. 6, when $z_{B}$ exceeds $z_{\alpha}$ by a factor of five, even in the region of maximum phonon energy density, the energy density is much less than its initial value. Under the conditions of the experiments considered, the intensity was 0.055 of its initial value.

Figure 3 shows the temporal dependence of signal amplitude for a definite value of temperature $T_{0}=1.0 \mathrm{~K}$ in the initial Bose-Einstein distribution function. The plots in the Fig. 3 are obtained from the formula (7) with the numerical values of the parameters given above. Figure 3 shows that the half width of the pulse broadening due to dispersion is close to (90), and the contribution of the angular width is negligible, in accordance with the result obtained from Eq. (74).

Estimations of the temperatures of the heater in the experiments [16] was $>1 \mathrm{~K}$ for heater powers $>0.3 \mathrm{~mW}$. However, the theoretical length of a pulse at $T=1 \mathrm{~K} \mathrm{ex}^{-}$ ceed the observed ones for heater powers $>0.3 \mathrm{~mW}$. The theoretical values of the increase in pulse length, at different initial temperatures (see [16]) agree with those calculated here, for initial temperatures in the interval $0.2-0.8 \mathrm{~K}$. The same results were found in [16] using a computer calculation. These calculations were made using the expression in [16], which describes the ballistic motion of phonons moving strictly parallel to the $z$ axis, with group velocities given by the dispersion law (87). 


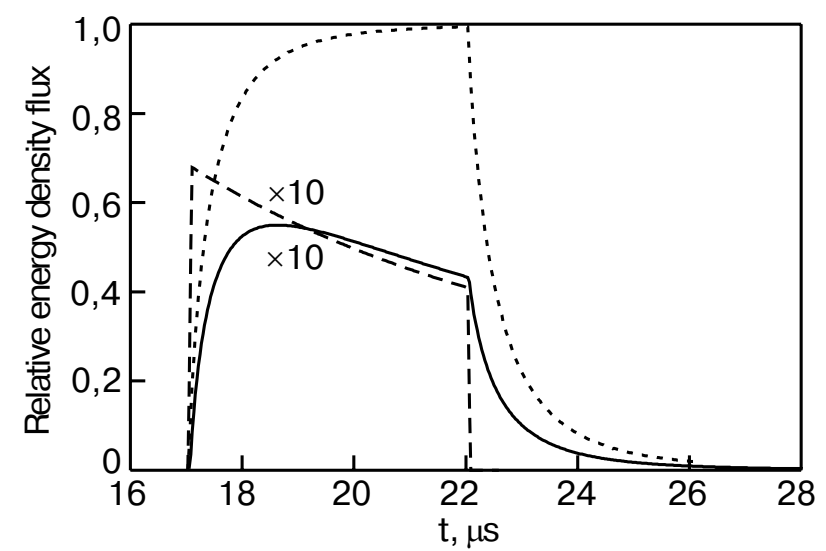

Fig. 3. The temporal dependence of the energy density flux at $z=8 \mathrm{~mm}, t_{p}=5 \mu s, \theta_{p} \approx 17.6^{\circ}$, and $L_{\perp}=0.5 \mathrm{~mm}$, relative to that of the initial pulse. The initial temperature of the pulse is $T_{0}=1.0 \mathrm{~K}$. The dotted line represents the signal when there is only dispersion, the dashed line represents the signal without dispersion but with a finite angular width in the initial pulse, and the solid line represents the signal with both dispersion and finite angular width. Note that the dashed and solid lines have had their $y$ values multiplied by 10 .

This model phonon pulse changes its form just because of dispersion.

The conclusions from both analyses is that the temperature of the initial pulse of phonons is much lower than the temperature of the heater which creates them.

According to the experimental conditions in [16] angular spreading does not contribute to the increase of the length of the pulse. However, the finite value of angle $\theta_{p}$ determines the transverse spreading of the pulse. Under the experimental conditions [16], this spreading results in a considerable decrease of the amplitude of the signal along the axis of the pulse. Thus, the amplitude of the pulse at the detector is a factor 0.055 smaller than the amplitude of the initial pulse.

Undoubtedly, a series of experiments at high pressure similar to experiments at the saturated vapor pressure [13] are of great interest. This would give the temporal and spatial dependence of the phonon energy density for different pulse lengths. The comparison of results of such experiments at high pressure and saturated vapor pressure would make it possible to com- pare the observed values with those calculated with the model of strongly interacting phonons (see [14]) and the results obtained here for a system of phonons without interactions. This comparison will give us important information about anisotropic systems of phonons in superfluid helium.

We express our gratitude to EPSRC of the UK (grant GR/S24855 and GR/N20225), and to GFFI of Ukraine (grant N02.07/000372) for support for this work.

1. A.F.G. Wyatt, N.A. Lockerbie, and R.A. Sherlock, Phys. Rev. Lett. 33, 1425 (1974).

2. M.J. Baird, F.R. Hope, and A.F.G. Wyatt, Nature 304, 325 (1982).

3. F.R. Hope, M.J. Baird, and A.F.G. Wyatt, Phys. Rev. Lett. 52, 1528 (1984)

4. M. Brown and A.F.G. Wyatt, J. Phys.: Condens. Matter 2, 5025 (1990)

5. M.A.H. Tucker and A.F.G. Wyatt, Science 283, 1150 (1999)

6. H.J. Marris and W.E. Massey, Phys. Rev. Lett. 25, 220 (1970)

7. M.A.H. Tucker and A.F.G. Wyatt, J. Phys.: Condens. Matter 6, 2813 (1994).

8. M.A.H. Tucker and A.F.G. Wyatt, J. Phys.: Condens. Matter 6, 2825 (1994).

9. I.N. Adamenko, K.E. Nemchenko, A.V. Zhukov, M.A.H. Tucker, and A.F.G. Wyatt, Phys. Rev. Lett. 82, 1482 (1999).

10. A.F.G. Wyatt, M.A.H. Tucker, I.N. Adamenko, K.E. Nemchenko, and A.V. Zhukov, Phys. Rev. B62, 9402 (2000)

11. I.N. Adamenko, K.E. Nemchenko, and A.F.G. Wyatt, Fiz. Nizk. Temp. 28, 123 (2002) [Low Temp. Phys. 28, $85(2002)]$.

12. M.A.H. Tucker and A.F.G. Wyatt, J. Low Temp. Phys. 113, 621 (1998).

13. R.V. Vovk, C.D.H. Williams, and A.F.G. Wyatt, Phys. Rev. B68, 134508 (2003).

14. I.N. Adamenko, K.E. Nemchenko, V.A. Slipko, A.F.G. Wyatt, Phys. Rev. B68, 134507 (2003).

15. R.C. Dynes and V. Narayanamurti, Phys. Rev. Lett. 33, 1195 (1974)

16. A.F.G. Wyatt, R.A. Sherlock, and D.R. Allum, J. Phys.: Condens. Matter 15, 1897 (1982).

17. A.F.G. Wyatt and D.R. Allum, J. Phys.: Condens. Matter 15, 1917 (1982).

18. I.M. Khalatnikov, An Introduction to the Theory of Superfluidity, Addison-Wesley Publishing, New York (1988).

19. I.M. Khalatnikov, Zh. Eksp. i Teor. Fiz. 22, 687 (1952) (in Russian).

20. N.A. Lockerbie, Ph. D. Thesis, Nottingham University (1975) 